\title{
Microfluidic magnetic fluidized bed for DNA analysis in continuous flow mode
}

Iván Hernández-Neuta ${ }^{*}$, lago Pereiro ${ }^{b, c, d *}$, Annika Ahlforda ${ }^{2}$ Davide Ferraro ${ }^{b, c, d}$, Qiongdi Zhang ${ }^{b, c, d}$, Jean-Louis Viovy ${ }^{b, c, d}$, Stéphanie Descroix ${ }^{b, c, d * *}$ and Mats Nilsson ${ }^{a * *}$

a Science for Life Laboratory, Department of Biochemistry and Biophysics, Stockholm University, Stockholm Sweden, Tomtebodavägen 23B, SE-171 65, Solna, Sweden

b Laboratoire Physico Chimie Curie, Institut Curie, PSL Research University, CNRS UMR168, 75005, Paris, France

c Sorbonne Universités, UPMC Univ Paris 06, 75005, Paris, France

d Institut Pierre-Gilles de Gennes, 75005, Paris, France

\author{
* Authors contributed equally to this work \\ ** Corresponding authors
}

Key words: Magnetic beads, microfluidic fluidized bed, DNA analysis, Padlock probes, rolling circle amplification

\begin{abstract}
Magnetic solid phase substrates for biomolecule manipulation have become a valuable tool for simplification and automation of molecular biology protocols. However, the handling of magnetic particles inside microfluidic chips for miniaturized assays is often challenging due to inefficient mixing, aggregation, and the advanced instrumentation required for effective actuation. Here, we describe the use of a microfluidic magnetic fluidized bed approach that enables dynamic, highly efficient and simplified magnetic bead actuation for DNA processing in a continuous flow platform with minimal technical requirements. We evaluate the performance of this approach by testing the efficiency of individual steps of a DNA assay based on padlock probes and rolling circle amplification (RCA). This assay comprises common nucleic acid analysis principles, such as hybridization, ligation, amplification and restriction digestion. We obtained efficiencies of up to $90 \%$ for these reactions and high throughput capabilities, with flow rates up to $5 \mu \mathrm{L} / \mathrm{min}$ without compromising performance. The obtained efficiency values using the fluidized bed were superior to a commercially available solution for microfluidic manipulation of magnetic beads. Moreover, to demonstrate the potential of this approach for integration into micro-total analysis systems, we optimized the production of a low-cost polymer based microarray and tested its analytical performance for integrated single-molecule digital read-out. Finally, we provide the proof-of-concept for a single-chamber microfluidic chip that combines the fluidized bed with the polymer microarray for a highly simplified and integrated magnetic bead-based DNA analyzer, with potential applications in diagnostic systems.
\end{abstract}




\section{Introduction}

Microfluidics has become an increasingly important toolbox for automation and miniaturization of chemical and biochemical assays, allowing to simplify the execution of complex protocols in miniaturized chips or micro-total analysis systems ( $\mu$ TAS) (Sackmann et al. 2014) for research and diagnostics. Low Reynolds number regimes allow the manipulation of liquid reagents with high precision in a cost effective way (Duncombe et al. 2015). However, this laminar flow regime severely hinders mixing of solutions, hence restricting the adaptation of biochemical processes where sequential exchange and optimal mixing of multiple reagents are required. Biomolecule immobilization on surfaces has facilitated this exchange in both microfluidic and bulk operations, but in the case of flat surfaces large diffusion lengths often translate into either time consuming or suboptimal mass transfer to the substrate (Squires et al. 2008). Attempts to overcome this limitation has led to research on systems presenting higher surface to volume ratios, including patterned substrates, porous monoliths or particles. Of these, magnetic microparticles or beads offer possibly the most advantageous characteristics, as they largely simplify chip architectures, allow distance manipulation, present high functionalization versatility, are commercially available and cost efficient (Gijs et al. 2010). The manipulation of magnetic beads in microfluidic channels is commonly achieved by using electro-magnets (Ferraro et al. 2016), coils (Teste et al. 2015), or permanent magnets (Hoshino et al. 2011). Electrical actuation or physical displacement allows rapping and release of the magnetic beads on demand for specific extraction, washing and reagent exchange. Notably, under exposure to an external magnetic field, magnetic beads form supramolecular structures in the form of microcolumns due to dipole-dipole interactions. This property has been exploited to optimize perfusion with liquids (Lacharme et al. 2008) and has proven particularly useful for the capture of larger entities such as cells (Hayes et al. 2001; Saliba et al. 2010). However, in the absence of geometrical constraints the columns tend to laterally interact to form larger bead ensembles with limited percolation capacity (Le Nel et al. 2008). Moreover, the constant contact of biofuntionalized beads often leads to the irreversible formation of aggregates that may cause inhibition of reactions in which sequential exchange of reagents is required (Gijs 2004; Hayes et al. 2001; Rida and Gijs 2004). A dynamic manipulation of magnetic beads can effectively reduce this problem, as shown by digital microfluidics (Wang et al. 2007) and ultrasound acoustics (Iranmanesh et al. 2016; Yaralioglu et al. 2004). However, these methods still require relatively complex fabrication and operation protocols, particularly when performing multi-step bioassays.

The use of magnetic beads for nucleic acid manipulations has enabled the development of wellestablished protocols for simplified extraction, purification (Berensmeier 2006), amplification (Kojima et al. 2005) and detection (Tamanaha et al. 2008), including multiplex suspension arrays and next generation sequencing technologies (Margulies et al. 2005). In recent years, padlock probes (PLP) (Nilsson et al. 1994) and rolling circle amplification (RCA)-based reactions, have proven excellent nucleic acid analytical tools for in vitro (Dahl et al. 2004; Jarvius et al. 2006) and in situ assays (Larsson 
et al. 2004; Lizardi et al. 1998). PLPs allow for specific and stringent recognition of multiple nucleic acid sequences, due to the combination of hybridization and ligation chemistries in a single bimolecular reaction step, to form independent and target-specific circular DNA molecules. Reacted probes serve as substrates for RCA, an isothermal single-molecule amplification process that produces long DNA concatemers (rolling circle products - RCPs) of sub-micron size that can be quantified in a digital manner (Jarvius et al. 2006; Lizardi et al. 1998). The combination of these principles led to the development of a versatile assay for nucleic acid analysis, which includes multiple advantages such as single nucleotide resolution, isothermal amplification and single-molecule digital quantification with high multiplexing capability (Hardenbol et al. 2005). The use of magnetic beads to perform a PLP/RCA - based assay has been reported for circle-to-circle amplification (C2CA) (Dahl et al. 2004) in which, for an increased amplification performance, RCPs are restriction digested, monomers are circularized and a second round of RCA is performed to increase the assay's analytical sensitivity. The inclusion of magnetic beads provided a solid phase for washing and reagent exchange, with high-surface to volume ratio for optimal reaction conditions (Goransson et al. 2012). It allowed for decreased assay time, as higher concentrations of PLPs could be used to enhance reaction kinetics and PLP ligation could be performed in minutes instead of hours. Magnetic bead-based PLP/C2CA has proven suitable for the detection of a number of infectious diseases (Engstrom et al. 2013; Ke et al. 2011; Mezger et al. 2015a; Mezger et al. 2015b; Mezger et al. 2014), but despite its demonstrated performance regarding specificity and sensitivity, this assay is composed of a complex multi-step protocol that requires handling and exchange of various reagents, technical expertise, and a dedicated instrument for read-out. This makes it unattractive for wider use in research laboratories, and challenging for adaptation to diagnostic settings. To date, the only attempt to automate and simplify magnetic bead-based PLP/C2CA using microfluidics has been reported by Kühnemund et al. (Kuhnemund et al. 2014), who described the design and testing of a digital microfluidic chip for this assay in a semi-automated fashion. However, the operations were restricted to (i) low sample $(1.25 \mu \mathrm{L})$ and reagent volumes, limiting throughput, (ii) a relatively complex and costly platform, and (iii) a read out that required off-chip digital quantification on a microscope slide.

In this work, we report the adaptation of a microfluidic magnetic bead mixing approach for DNA analysis using PLPs and C2CA. This simple passive magnetic bead mixing approach is based on a fluidized bed principle that generates a constant hydrodynamic recirculation of magnetic beads, contained in a geometrically simple microfluidic chamber in a continuous-flow mode (Pereiro et al. 2017a; Pereiro et al. 2017b). The use of this principle allows for efficient bead-liquid mixing and optimal perfusion of incoming liquids inside the microfluidic structures. The internal recirculation and reduced diffusion lengths maximize analyte interaction with the surface in a continuous flow-through mode. We implemented this approach to perform sequential nucleic acid manipulation reactions comprised in a PLP/C2CA-based protocol, including: hybridization, ligation, amplification and restriction digestion. We compared the fluidized bed to other magnetic bead handling approaches for continuous/stop-flow microfluidics, and found it to perform better than the others. We demonstrate the utility of this principle for incorporation into $\mu$ TAS, for which we designed PLPs that contained a universal backbone sequence with a stem-hairpin structure (Lohmann et al. 2007). This special design enables a surface transfer based amplification, and allows to perform a C2CA reaction in which each of the two amplification steps 
are conducted on a dedicated solid surface: a 3D magnetic bead surface for efficient heterogeneous reactions, and a 2D planar surface for localized digital quantification read-out with standard fluorescence microscopy. For the latter, we show the adaptation and optimization of a low cost polymer-based microarray production approach (Sun et al. 2012) for the detection of RCPs, and demonstrate its analytical capabilities for detecting a mutation in the oncogene KRAS. Finally, with the established conditions, we designed an integrated $\mathrm{COC}$ microfluidic chip that combined the fluidized bed with the polymer microarray in a single chamber, and demonstrated the proof-of-concept for its use as a microfluidic DNA analyzer, achieving integration from target capture to read-out in a simple, singlechamber continuous-flow microfluidic chip.

\section{Materials and methods}

Superparamagnetic MyOne dynabeads ${ }^{\circledR}$ T1 with streptavidin surface functionalization were acquired from Thermofisher (Stockholm, Sweden). Oligonucleotides used in this study (Table 1) were synthetized by Integrated DNA Technologies (Heverlee, Belgium). Amp ligase enzyme and 10x Amp ligase buffer were purchased from Epicentre (Nordic Biolabs AB, Täby, Sweden). Phi 29 polymerase and phi29 10X reaction buffer were obtained from Thermo Scientific (Göteborg, Sweden) and New England Biolabs

(Évry, France). T4 DNA ligase, dNTP mix and ATP were purchased from DNA Gdansk (Gdansk, Poland). BSA, Sapl and Alul restriction enzymes were obtained from New England Biolabs (Bionordika, Stockholm, Sweden). Tris-HCl (pH 7.5), $\mathrm{NaCl}$, EDTA and PBS were purchased from Karolinska Universitetslaboratoriet (Stockholm, Sweden). Hexadecane, isopropanol, SCC, SDS, Triton X Tween20 were obtained from Sigma-Aldrich AB (Stockholm, Sweden) and Cyclic olefin co-polymer (COC) Topas ${ }^{\circledR}$ slides were acquired from TOPAS Advanced Polymers GmbH (Frankfurt-Höchst, Germany) and poly(dimethylsiloxane) (PDMS) Sylgard $\AA 184$ silicone elastomer kit from Dow Corning (Ellsworth Adhesives France, Pontoise, France).

\subsection{Microfluidic chip fabrication}

PDMS chips used for efficiency tests were manufactured as previously described (Pereiro et al. 2017a). The design is illustrated in Fig. 1a and comprised an optimized rhombic chamber geometry (Pereiro et al. 2017b) with an aperture angle of $13^{\circ}$ and $25^{\circ}$ at the inlet and outlet respectively, $13 \mathrm{~mm}$ in length and $2.6 \mathrm{~mm}$ at its maximum width. The inlet channel was $100 \mu \mathrm{m}$ wide and all the structures $50 \mu \mathrm{m}$ high. The chips were fabricated by PDMS casting, pouring a 10:1 mix with the cross linker (Sylgard® 184, Dow Corning) on top of a brass micro-milled mold. The cured PDMS layer with chamber structures were bonded immediately after plasma treatment onto a second flat PDMS film cast on a petri dish. The surface of the closed channels was treated by $1 \mathrm{~h}$ incubation with a $0.25 \%$ (w/v) poly-dimethylacrylamide bearing epoxy function (E-PDMA)1 aqueous solution, followed by rinsing with deionized water.

$\mathrm{COC}$ chips for integrated protocol tests were fabricated by hot embossing with the same brass micromilled molds at $120^{\circ} \mathrm{C}$ for 10 min under 5 bar of pressure. After inlet and outlet drilling, the embossed 
piece was closed with COC microarray slides through solvent bonding by applying $15 \%$ hexadecane and $75 \%$ isopropanol in the interface and pressing at $5 \mathrm{bar}, 70^{\circ} \mathrm{C}$ for $10 \mathrm{~min}$. Tygon $\odot$ tubes $(2 \mathrm{~mm})$ in tight contact with PEEK tubes (50 $\mu \mathrm{m}$ and $250 \mu \mathrm{m}$ for inlet and outlet internal diameters, respectively) were plugged inside the drilled holes to ensure complete sealing. The chip design was similar to the PDMS chip but was $3 \mathrm{~cm}$ in length, $5 \mathrm{~mm}$ at its maximum width, with an angle of $13^{\circ}$ at the inlet and $25^{\circ}$ at the outlet. The array was placed in the wider area of the chamber.

Table 1. Oligonucleotides used in this study. Blue: Target complementary regions. Purple: Hairpin-loop complementary sequence, Red: Ligation barcode split regions. Orange: Barcode oligonucleotide region. Green: Capture oligonucleotide region. Codon underlined correspond to the targeted KRAS codon 12.

\begin{tabular}{|c|c|}
\hline Name & Sequence (5' to $3^{\prime}$ end) \\
\hline PLP wild type & $\begin{array}{l}\mathrm{PO}_{4} \text { - } \underline{\mathrm{C} A G C T C C A A C T A C C A C A ~ C C T C A G T A A T A G T G T C T T A C ~ C T G T C A G A T T ~} \\
\text { GGAAGAGCCTTTGCTCTTCCAAT AGTGAGCATG TCTTGCCTACGCCAC }\end{array}$ \\
\hline PLP mutant & $\begin{array}{l}\mathrm{PO}_{4} \text { - } \text { CAGCTCCAACTACCAC GCCGCAGATG GGAAGAGCTTTTGCTCTTCCCAT } \\
\text { CAACACGATA AGCCAAACATGTGCATTGAG CTCTTGCCTACGCCAT }\end{array}$ \\
\hline $\begin{array}{l}\text { Capture } \\
\text { oligonucleotide }\end{array}$ & $\begin{array}{l}\text { Biotin - TCTCTCTCTCTCTCTCTCTCTCTC } \\
\text { TCATTTTCAGCAGGCCTTATAATAAAAATAATGAAAATGT }\end{array}$ \\
\hline $\begin{array}{l}\text { DNA target KRAS } \\
\text { wild type }\end{array}$ & $\begin{array}{l}\text { ACATTTTCATTATTTTTATTATAAGGCCTGCTGAAAATGACTGAATATAAACTTGTGGT } \\
\text { AGTTGGAGCT } \underline{\text { GTGGCGTAGGCAAGAGTGCCTTGACGATA }}\end{array}$ \\
\hline $\begin{array}{l}\text { DNA target KRAS } \\
\text { wild type - FITC }\end{array}$ & $\begin{array}{l}\text { ACATTTTCATTATTTTTATTATAAGGCCTGCTGAAAATGACTGAATATAAACTTGTGGT } \\
\text { AGTTGGAGCTGGTGGCGTAGGCAAGAGTGCCTTGACGATA - FITC }\end{array}$ \\
\hline $\begin{array}{l}\text { DNA target KRAS } \\
\text { mutant }\end{array}$ & $\begin{array}{l}\text { ACATTTTCATTATTTTTATTATAAGGCCTGCTGAAAATGACTGAATATAAACTTGTGGT } \\
\text { AGTTGGAGCTGATGGCGTAGGCAAGAGTGCCTTGACGATA }\end{array}$ \\
\hline $\begin{array}{l}\text { Ligation barcode } \\
\text { wild type }\end{array}$ & СтСтСтСтстСтСтСтСтСт CTGTCAGATTAGTGAGC*A*T*G \\
\hline $\begin{array}{l}\text { Ligation barcode } \\
\text { mutant }\end{array}$ & 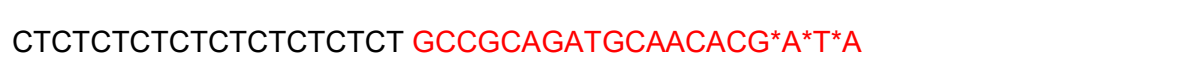 \\
\hline $\begin{array}{l}\text { Detection oligo } \\
\text { wild type }\end{array}$ & Су3 - TTTTTGTAAGACACTATTACTGAGG \\
\hline $\begin{array}{l}\text { Detection oligo } \\
\text { mutant }\end{array}$ & Cy5 - CTCAATGCACATGTTTGGCT \\
\hline
\end{tabular}

${ }^{*}$ Phosphorothioate bonds

\subsection{Microarray printing}

The microarray printing procedure using direct UV DNA oligonucleotide grafting on the polymer surface, was adapted from Sun et al. (Sun et al. 2012). For this, $1 \mathrm{~mm}$ thick COC slides (TOPAS $®$ ) were washed with isopropanol and sonicated. Washed foils were irradiated with UV light at a wavelength of $254 \mathrm{~nm}$, for $30 \mathrm{~s}$ at a power of $10 \mathrm{~mW} / \mathrm{cm}^{2}$. Ligation barcode oligonucleotides were diluted to $10 \mu \mathrm{M}$ in $150 \mathrm{nM}$ sodium phosphate buffer with $0.004 \%$ Triton-X 100 and $50 \mathrm{~nL}$ droplets were automatically pipetted onto the irradiated foils with a Rotaxis (Cetoni $\mathrm{GmbH}$ ) positioning system controlled by the Qmix Element software. After 5 min incubation, the foils were washed with 0.1 X SCC containing $0.1 \%$ SDS for 10 min with rotation, rinsed with deionized water and dried.

\subsection{Microfluidic setup}

Fluidic handling was achieved with a pressure-based microfluidic flow control system (MFCS ${ }^{\text {TM }}$ Fluigent, France) with three independent pressure outlets. The flow controller was further feedback-regulated with 
flow rate measurements obtained at the exit of the chip by flow rate sensors (Type-S Flowell ${ }^{\mathrm{TM}}$, Fluigent). Pressure controller and flow-rate sensors were operated by the MAESFLO ${ }^{\mathrm{TM}}$ software. Temperature control for efficiency experiments was achieved with a thermoelectric element coupled to a heat sink and a fan for heat dissipation during cooling; the element was controlled with TEC software v2.10 (Meerstetter Engineering $\mathrm{GmbH}$, Rubigen, Switzerland). For the integrated chip experiments, an indium tin oxide coated glass slide connected to a voltage controller (Eurotherm 3508) was feedback-regulated with a thermocouple (placed between the chip and the slide).

For formation of the magnetic fluidized bed, the microfluidic chip was first primed with $1 \mathrm{X}$ washing buffer (10 mM Tris- $\mathrm{HCl}$ pH 7.5, $5 \mathrm{mM}$ EDTA, $100 \mathrm{mM} \mathrm{NaCl}, 0.1 \%$ Tween 20). Next, $10 \mu \mathrm{L}$ of functionalized magnetic bead suspension $(5 \mu \mathrm{g} / \mu \mathrm{L})$ were loaded through the outlet by removing the outlet tubing and inserting a pipette tip loaded with the magnetic bead solution. The beads were guided inside the chamber using a small magnet placed at the bottom of the chip. Once the beads were loaded inside the chamber, the outlet tubing was placed back and an NdFEB 1.47 T permanent magnet (N50, Chen yang Technologies) was positioned $2 \mathrm{~mm}$ from the bent inlet channel, aligned with the central axis of the microfluidic chamber.

For the efficiency comparison experiments, we used the ChipGenie ${ }^{8}$ edition $\mathrm{P}$ (Microfluidic Chipshop, Jena, Germany), a benchtop instrument that poses a linearly moving permanent magnet as well as temperature control. Microscope slide format $(75 \times 25 \mathrm{~mm})$ COC chips with single channels, $58.5 \mathrm{~mm}$ length $\times 2 \mathrm{~mm}$ width $\times 0.2 \mathrm{~mm}$ depth (Microfluidic Chipshop, Jena, Germany), were used and a syringe pump (New Era Pump Systems, Inc.) was used for fluidic handling. Dupont $₫$ FEP Tubing Natural 1/16" OD x .008" ID $(200 \mu \mathrm{m})$ was used for fluidic connections.

\subsection{Efficiency tests for target capture, PLP ligation, RCA and restriction digestion}

$5 \mu \mathrm{l}$ of $10 \mathrm{mg} / \mathrm{ml}$ T1 MyOne Dynabeads ${ }^{\circledR}$ suspension was washed with $20 \mu \mathrm{l} 1 \mathrm{X}$ washing buffer three times and functionalized by incubation with $250 \mathrm{nM}$ biotinylated capture oligonucleotide solution for 10 min in $1 \mathrm{X}$ binding buffer (10 mM Tris- $\mathrm{HCl} \mathrm{pH} 7.5,5 \mathrm{mM}$ EDTA, $1 \mathrm{M} \mathrm{NaCl}, 0.1 \%$ Tween 20), beads were washed and re-suspended in $1 \mathrm{X}$ binding buffer after incubation. Target capture efficiencies for different modes of magnetic manipulation were determined by flowing $80 \mu \mathrm{l}$ of a $50 \mathrm{nM}$ fluorescently-tagged target solution through the microfluidic chip with fluidized bed at $1 \mu \mathrm{L} / \mathrm{min}$, and comparing the average fluorescence intensity at the outlet channel with that obtained from a control without the fluidized bed. The efficacies at 3 and $5 \mu \mathrm{L} / \mathrm{min}$ were obtained similarly, but measuring the fluorescence intensity every 4 and 6 min respectively, until $120 \mu \mathrm{L}$ of the target solution was flowed. Measurements were performed using an epifluorescence microscope (Eclipse Ti-E, Nikon, Japan).

The efficiency of individual on-chip reaction steps was tested by comparison to the number of RCPs obtained in a control tube. Control tubes and off-chip reactions were performed as follows: Magnetic beads were functionalized as described above, and re-suspended in $2 \mathrm{X}$ binding buffer, target capture was performed by mixing $10 \mu \mathrm{L}, 100 \mathrm{fM}$ dilution of KRAS synthetic target oligonucleotide with $10 \mu \mathrm{L}$ of magnetic bead suspension and incubated at $60^{\circ} \mathrm{C}$ for $5 \mathrm{~min}$. Beads were collected with a magnet, the 
supernatant discarded and washed twice with $1 \mathrm{X}$ washing buffer. Subsequently, $20 \mu \mathrm{L}$ ligation mix containing $50 \mathrm{nM}$ PLPs, $0.2 \mu \mathrm{g} / \mu \mathrm{L}$ BSA, 1X Ampligase buffer and $0.25 \mathrm{U} / \mu \mathrm{L}$ Ampligase enzyme, was added to the beads, gently vortexed and incubated at $60^{\circ} \mathrm{C}$ for 5 minutes. After ligation, the supernatant was discarded and beads were re-suspended in $20 \mu \mathrm{L}$ RCA amplification mix containing: $0.2 \mu \mathrm{g} / \mu \mathrm{L}$ BSA, 1X phi 29 polymerase buffer, $125 \mu \mathrm{M}$ dNTPs and $200 \mathrm{mU} / \mu \mathrm{L}$ phi29 polymerase. Beads were then washed 3 times with washing buffer.

For simultaneous restriction digestion and ligation of monomers off-chip, the supernatant was removed and $30 \mu \mathrm{L}$ mix, containing $0.2 \mu \mathrm{g} / \mu \mathrm{L}$ BSA, 1X Phi29 polymerase buffer, $360 \mathrm{mU} / \mu \mathrm{L}$ Sapl restriction enzyme, $5 \mu \mathrm{M}$ Ligation oligonucleotide mix, $680 \mu \mathrm{M}$ ATP and $10 \mathrm{mU} / \mathrm{mL}$ T4 DNA ligase, was added and incubated for $10 \mathrm{~min}$ at $37^{\circ} \mathrm{C}$. Beads were discarded and the supernatant was recovered for the second $\mathrm{RCA}$ following the same conditions mentioned above. Final amplification products were fluorescently labeled by mixing the amplification solution with fluorescently tagged detection oligonucleotides in a final concentration of $5 \mathrm{nM}$ and $1 \mathrm{X}$ binding buffer. Labeling was achieved by incubation at $75^{\circ} \mathrm{C}$ for 2 min followed by hybridization at $55^{\circ} \mathrm{C}$ for $10 \mathrm{~min}$. Final amplification products in solution were quantified by amplified single molecule detection (ASMD) with a dedicated instrument, Aquila 400 (Q-Linea, Sweden).

For on-chip reactions, mixes were prepared at the same concentrations as stated above. After the magnetic fluidized bed was formed, reagents and mixes were prepared freshly and exchanged manually in the reservoir connected to the input channel. The flow rate for target capture, PLP ligation and RCP restriction/monomer ligation was $2 \mu \mathrm{L} / \mathrm{min}$, and RCA was performed at $1 \mu \mathrm{L} / \mathrm{min}$. In the experiments for calculating individual reaction efficiencies, single reaction steps were performed on-chip while the previous and remaining reaction steps were performed off-chip.

\subsection{Microarray characterization}

The microarray was characterized by preparing serial dilutions resulting in different ratios $(1: 1,1: 10$, 1:100 and 1:1000) of the KRAS wild type and mutant synthetic targets (Table 1). Target capture, PLP ligation and RCA was performed off-chip as described earlier. After washing twice with the binding buffer, beads were suspended in the ligation/restriction mix, added to the microarray and incubated for $15 \mathrm{~min}$ at $37^{\circ} \mathrm{C}$. Beads were discarded and the array was washed 3 times with washing buffer, $50 \mu \mathrm{L}$ of RCA mix was added to the microarray and incubated at the conditions described above. Labeling of arrayed RCA products was achieved using $50 \mathrm{nM}$ detection oligonucleotide in 1x binding buffer and incubating at $55^{\circ} \mathrm{C}$ for $20 \mathrm{~min}$. Arrays were again washed 3 times with washing buffer and imaged with an epifluorescence microscope (Axiolmager, Zeiss).

\subsection{Integrated chip tests}

For on-chip integrated experiments, the same concentrations and conditions as stated above were used for all the steps. The flow rates employed in different steps were as follows: Capture $5 \mu \mathrm{L} / \mathrm{min}$, PLP ligation $1 \mu \mathrm{L} / \mathrm{min}$, RCA $0.3 \mu \mathrm{L} / \mathrm{min}$, Restriction digestion/Ligation $0.5 \mu \mathrm{L} / \mathrm{min}$, Array $\mathrm{RCA} 0.3 \mu \mathrm{L} / \mathrm{min}$ and RCP labeling $1 \mu \mathrm{L} / \mathrm{min}$. 


\section{Results \& discussion}

\subsection{Microfluidic magnetic fluidized bed for nucleic acid analysis by C2CA}

The microfluidic chip is composed of a rhombic microchamber filled with a magnetic microbead suspension ( $1 \mu \mathrm{m}$ in diameter) (Fig. 1a). A permanent magnet, positioned at the inlet side of the chamber in alignment with the chamber's axis of symmetry, creates a magnetic field gradient that counteracts the dragging force exerted by the continuous-flow of incoming liquids. When these forces are in global equilibrium, the fluidized bed regime is established and beads recirculate dynamically by following the flow in the central region of the chamber and reversing direction once displaced towards the chamber sides, at the front of the bed (Pereiro et al. 2017b).

We applied this concept to perform a PLP/C2CA based assay for detection of DNA target sequences (Fig 1b). The assay comprises multiple sequential reaction steps. In the first step, streptavidin-coated magnetic beads were functionalized with biotinylated oligonucleotides partially complementary to the DNA sequence of interest (Table 1). The captured DNA target serves as a template for the circularization by hybridization and ligation of PLPs that have complementary target sequences at the 5' and 3' ends. For this study, PLPs were designed to contain a specific detection barcode, a ligation dual-barcode sequences and a stem-hairpin structure that contains the restriction cassette for Sapl endonuclease. Circularized PLPs were amplified by RCA, producing RCPs that contain multiple complementary copies of the labeling barcode, the ligation dual-barcode and the stem-hairpin. These $1^{\text {st }}$ generation RCPs were monomerized by restriction digestion using Sapl endonuclease and resulting RCP monomers were then released from the beads, circularized on the ligation barcodes and amplified by a second round of RCA (Fig. 1c). This produces $2^{\text {nd }}$ generation RCPs that were labeled with fluorescently-tagged complementary barcodes and digitally quantified in a microfluidic channel by amplified single molecule detection (ASMD) (Goransson et al. 2012; Jarvius et al. 2006) with a dedicated instrument. 

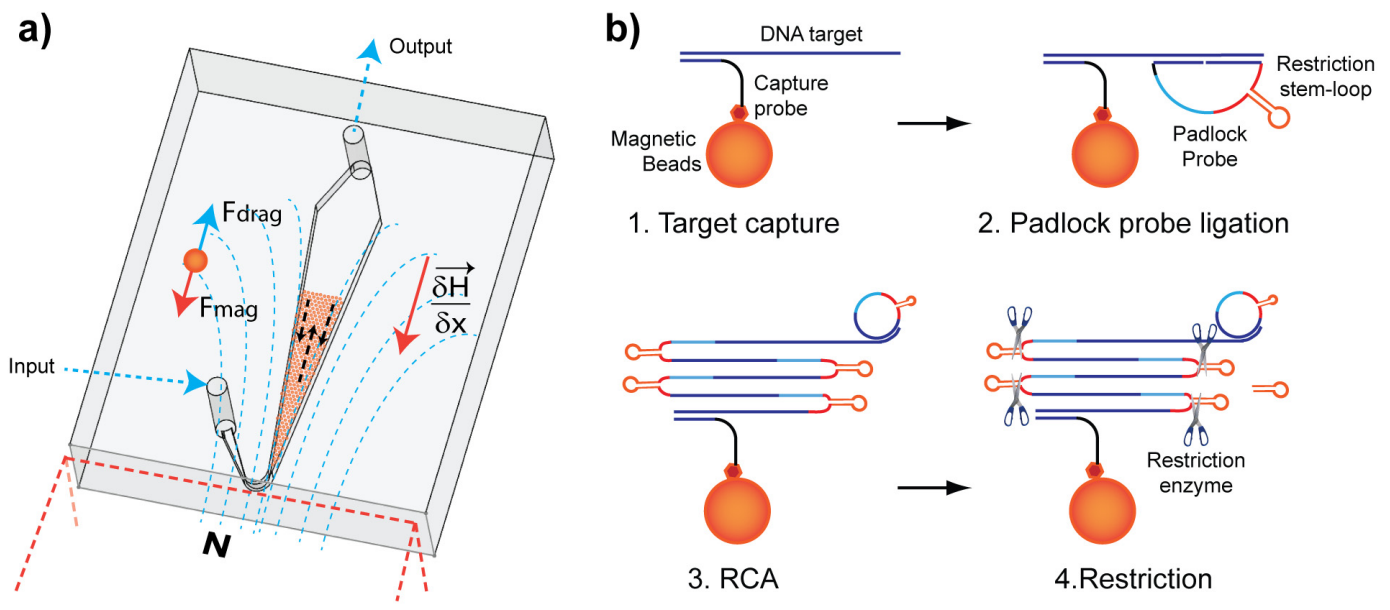

2. Target capture $\quad$ Padlock probe ligation

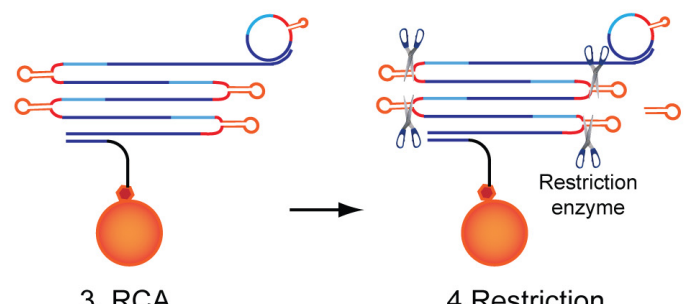

c)

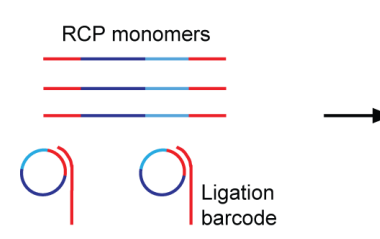

5. Monomer ligation

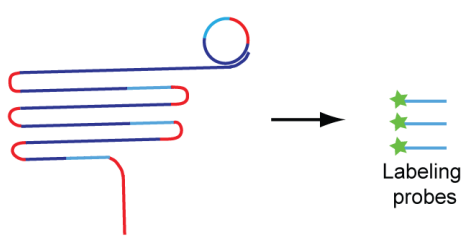

6. 2nd RCA

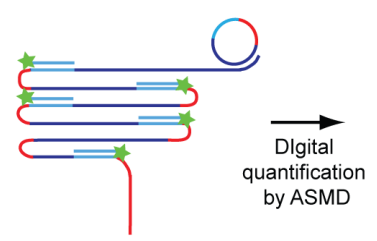

7. Labeling

Fig.1: Microfluidic magnetic fluidized bed for PLP/C2CA-based DNA analysis. a) Illustration of the microfluidic chip for magnetic fluidized bed. A dynamic recirculation of beads (indicated by dashed black arrows) in continuous flow is achieved by a balance of magnetic forces $\left(F_{\text {mag }}\right)$ exerted by the magnetic field gradient created by a permanent magnet positioned at the input of the chamber, and drag forces ( $\left.F_{\text {drag }}\right)$ exerted by the incoming liquid flow. b) Schematic of magnetic bead-based C2CA assay for nucleic acid analysis. 1. A DNA target sequence of interest is captured by hybridization, using magnetic beads functionalized with a capture probe that is partially complementary to the target of interest. 2. PLPs containing 5' and 3' ends complementary to the target (blue), a labeling barcode (light blue), ligation barcode split sequences (red) and a restriction stem-loop (orange), are hybridized and ligated by a thermostable DNA ligase. 3. Circularized probes are amplified by RCA, producing a long single stranded DNA concatemer containing multiple sequence complementary copies of the PLP. 4. RCPs are released from the beads by restriction digestion using an endonuclease targeting the ends of the stem-loop. c) RCP monomers are recircularized on the ligation barcodes, amplified by a $2^{\text {nd }}$ RCA and labeled with fluorophore-tagged barcodes for digital quantification by amplified single-molecule detection (ASMD) in homogenous solution

\subsection{Microfluidic magnetic fluidized bed facilitates efficient hybridization reactions}

In order to evaluate the efficiency of the microfluidic fluidized bed for capturing DNA strands based on hybridization chemistry (Fig 1b, Step 1.), magnetic beads were functionalized with the capture oligonucleotide, and a $50 \mathrm{nM}$ solution of a fluorescently-tagged DNA target was flowed through the chip. The average fluorescence intensity of the solution at the output of the chamber was measured, and the efficiency was calculated by comparing the fluorescence intensity values to a control experiment in which the DNA solution was flowed through the chip without the fluidized bed. We performed similar experiments using conventional rotating and static magnetic plug modes, in which the magnetic field was applied in a direction perpendicular to the liquid flow. Results are summarized in Fig. 2a: the highest capture efficiency was observed with the fluidized bed, indicating that the constant magnetic bead re- 
circulation, combined with the high bead density provide optimal conditions for fast hybridization kinetics. Since the DNA target solution is perfused efficiently through the bead suspension, transport of molecules to the active surface of the beads is facilitated. Moreover, the dynamic regime of the fluidized bed also contributes to avoiding local surface saturation effects, as the beads are in continuous recirculation within the incoming liquid. This is not the case for the static and rotating plugs where perfusion of the incoming solution through the plug is limited. As a consequence, the percolation of the incoming sample was limited to the plug exterior and to preferential paths internally formed, where the velocity of the liquid and diffusion lengths were more optimal, thus reducing effective transport to the active bead surfaces. These results show the importance of the dynamic regime necessary for optimal hybridization reactions.

a)

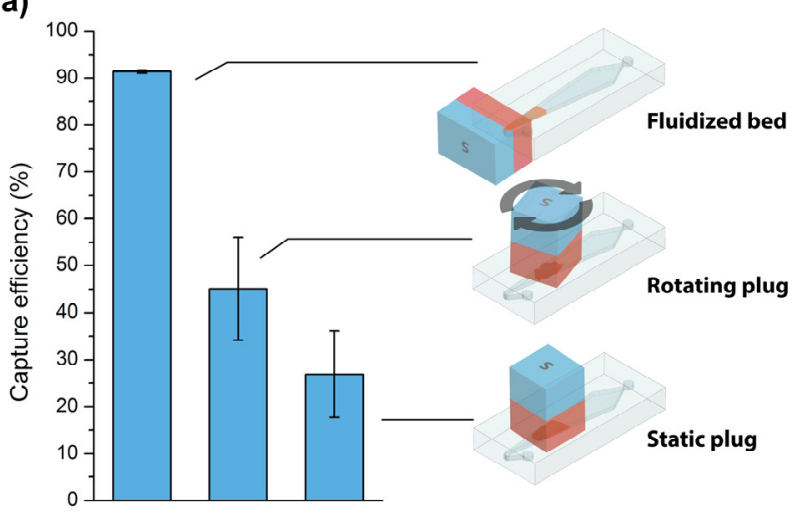

b)

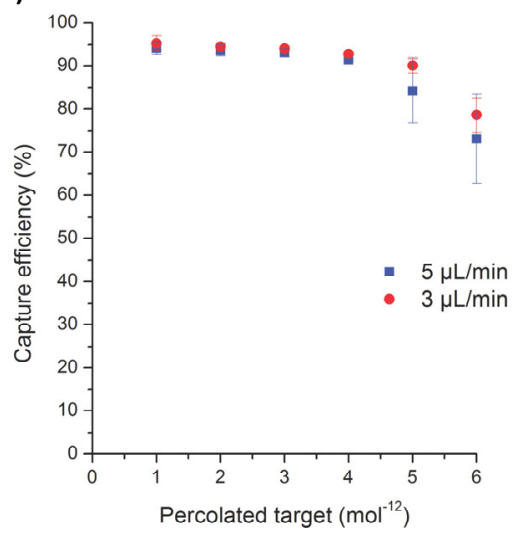

Fig. 2: Efficiency of high throughput hybridization reactions enabled by the fluidized bed. a) Figure showing DNA target capture efficiency for three different bead control regimes for $4 \mathrm{pmol}$ of percolated ssDNA at $1 \mu \mathrm{L} / \mathrm{min}$. A fluorescently labeled target solution is flowed through the microfluidic chip, and the fluorescence intensity is measured at the output of the chamber. The fluidized bed efficiency is compared to: (i) rotating plug, a magnet is rotated mechanically on top of the microchamber during target capture and (ii) static plug, a magnet is held in a permanent position perpendicular to the flow direction. Capture efficiency is calculated by comparing average fluorescence intensity values to a control without the fluidized bed. Error bars indicate standard deviation $(n=3) . \mathbf{b})$ DNA target capture efficiency of the microfluidic fluidized bed, using high flow rates. The efficiency was determined similarly as described for a) but measuring the fluorecence intensity at a number of time points while flowing 6 pmol of target, Error bars indicate standard deviation $(n=3)$.

Moreover, we evaluated the binding capacity of the fluidized bed by measuring the capture efficiency at high flow rates. Obtained results are illustrated in Fig. 2b: a capture efficiency of $93 \%$ was observed for up to $4 \mathrm{pmol}$ percolated target using flow rates at 3 and $5 \mu \mathrm{L} / \mathrm{min}$. Interestingly, the efficiency is maintained at both flow rate values, indicating that the capture is not limited by the residence time but only by the maximum binding capacity of the beads $(\sim 5 \mathrm{pmol})$ and therefore high sample volumes can be processed at a high capture rate $(\sim 70 \mathrm{fmol} / \mathrm{s})$. This result reinforces the observation of the previous experiment, indicating that when using the fluidized bed, the constant re-circulation of the beads in combination with the continuous flow allow to overcome diffusion barriers, improving mass transport and making hybridization reactions highly efficient. 
Additionally, the possibility of operating the magnetic plug at high flow rates enables high throughput processing as potentially $300 \mu \mathrm{L}$ of DNA solution can be processed in $1 \mathrm{~h}$ without any significant loss in capture efficiency. This possibly facilitates the analysis of large volumes of highly diluted extracts, which would be challenging for platforms like digital or droplet microfluidics, which are restricted to the analysis of small volumes per assay.

\subsection{Efficiencies of individual PLP/C2CA steps using the magnetic microfluidic fluidized bed}

We evaluated the microfluidic fluidized bed for performing individual steps of the magnetic bead PLP/C2CA protocol for DNA analysis (Fig. 1b), and compared it to ChipGenie ${ }^{\circledR}$ edition P (Fig 3), a commercially available benchtop solution for magnetic bead handling (Holubova et al. 2014; Julich et al. 2016; Julich et al. 2014; Kucerova et al. 2014). This instrument is designed with a holder for microscope slide format microfluidic chips, possesses a heating element and a magnet placed underneath the chip, and can perform magnetic bead actuation for mixing and incubation by mechanical movement of the magnet along the length of the microchannel. Efficiencies of individual steps on beads (capture, PLP ligation, RCA and digestion) (Fig. 1b, Steps 1-4) were evaluated using the microfluidic fluidized bed and the ChipGenie ${ }^{\circledR} \mathrm{P}$, comparing the final results to off-chip controls performed in regular PCR tubes that were used as $100 \%$ efficiency values for calculation. For this comparison, single reaction steps were performed on-chip and after completion, beads were recovered from the chips, and subsequent reactions were performed off-chip and quantified by ASMD. The results, summarized in Fig. 3 , indicate that the microfluidic fluidized bed displayed up to $50 \%$ higher efficiency than the ChipGenie $®$ $P$, and comparable results to those reported using digital microfluidics (Kuhnemund et al. 2014). This $50 \%$ efficiency increase of the fluidized bed compared to the ChipGenie for reactions such as RCA and restriction digestion, support the hypothesis that the hydrodynamic mode of the fluidized bed allows constant perfusion of incoming solutions and recirculation of the beads, which contributes to efficient mixing, thus making the surface of the beads highly available for reactions. In contrast, when using the ChipGenie $\circledast \mathrm{P}$, magnetic beads are concentrated in less than $4 \%$ of the total volume of the microfluidic channel. Hence, in spite of the mixing provided by the automated movement along the length of the channel, our results indicate that this mode of actuation is insufficient to achieve optimal contact between the beads and the bulk solution, affecting the performance of hybridization and enzymatic steps, probably due to local surface saturation. These results demonstrate that the use of microfluidic fluidized beds allows to overcome this limitation. The continuous-flow mode at which the fluidized bed is operated, as well as the smaller size of the microfluidic chip, further contribute to optimal interaction between the fluids and the solid phase as diffusion distances are minimized, in comparison to the microscope slide sized microfluidic chip used in the ChipGenie ${ }^{\circledR}$ P.

It was observed that decreased efficiencies for RCA and restriction digestion reactions were obtained compared to tube controls, using both the fluidized bed and the ChipGenie ${ }^{\circledR}$ P. Irreversible aggregation of the beads was observed while performing these steps. We hypothesize that this effect reduced the available surface and, in the case of the fluidized bed, hindered the recirculation of beads. The formation of the aggregates was also observed in control tubes, including those without DNA target (Suppl. Fig.1a), where the beads are not exposed to the magnet during the incubation times, suggesting that 
this phenomena is not caused solely by the constant exposure to the magnetic field. To investigate this, we incubated the beads at $37^{\circ} \mathrm{C}$ during $1 \mathrm{~h}$, in the RCA buffer, in absence of DNA, BSA or enzymes. Clusters were not observed using these conditions (Suppl. Fig.1a), indicating that the presence of these biomolecules might also play a role in aggregation. Additionally, we observed beads sticking to the surface of the microfluidic chips (PDMS and COC chip material), suggesting that unspecific hydrophobic interactions could also contribute to this phenomena. Interestingly, the size of the clusters was bigger when using the ChipGenie ${ } \mathrm{P}$ (Suppl. Fig.1b), which inversely correlates with the efficiency values obtained. This might imply that the dynamic mode of the fluidized bed also helps to avoid the clustering to some extent, and therefore aids to improved efficiencies.

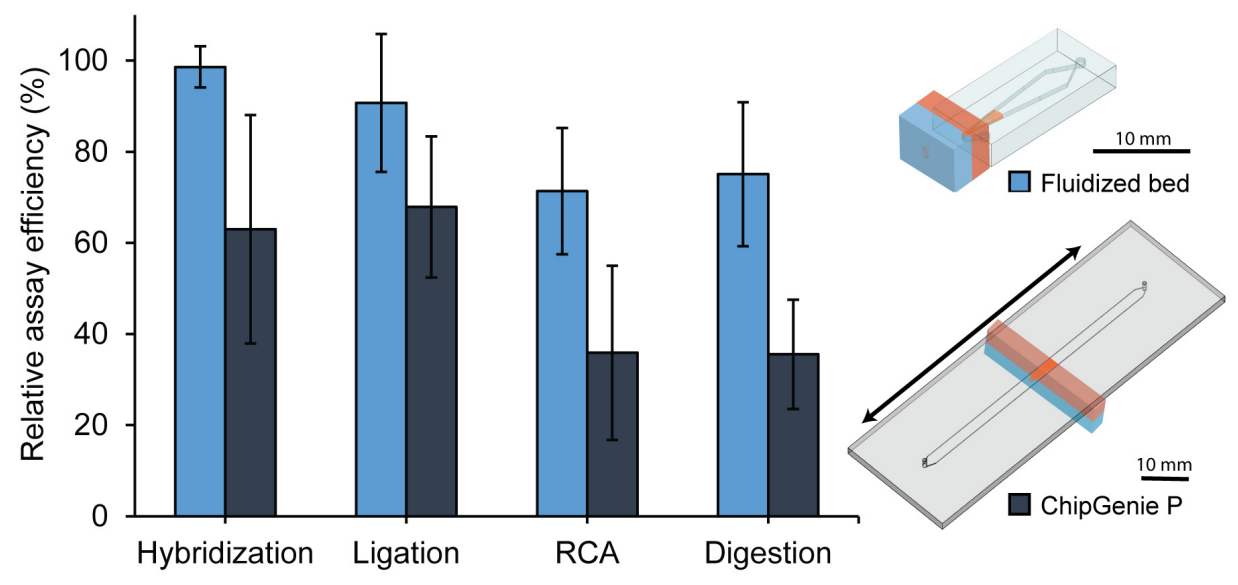

Fig.3. Efficiencies of individual C2CA steps in the microfluidic fluidized bed. Indicated steps were performed onchip either using the fluidized bed (upper right) or the ChipGenie $®$ edition $\mathrm{P}$ (bottom right), beads were recovered thereafter and subsequent steps were performed off-chip. RCP signals were compared to those obtained in control tubes (off-chip) and efficiencies were calculated. Bars indicate standard deviation $(n=3)$. Bottom right, Illustration of the ChipGenie ${ }^{\circledR} \mathrm{P}$ microfluidic chip and its operation principle for magnetic actuation in stop-flow mode. A microscope slide format $(75 \times 25 \mathrm{~mm})$ COC microfluidic chip with a single channel was used. The magnet was mechanically moved along the length of the channel to achieve magnetic mixing during incubation steps.

\subsection{Low cost polymer microarray for integrated digital read-out}

In the next step we tested the utility and versatility of the microfluidic fluidized bed as an effective module to miniaturize and integrate a complete PLP/C2CA assay in continuous-flow for digital DNA analysis. We first enabled the generation of $2^{\text {nd }}$ generation RCPs on an independent surface, so that RCP monomers after cleavage of $1^{\text {st }}$ generation RCPs (Fig 1b. Step 4) could be circularized on ligation barcodes grafted on this solid support, similar to the approach reported by Ericsson et al. (Ericsson et al. 2008). To achieve this, we designed and used PLPs comprising a complementary stem-hairpin structure, containing the restriction site of Sapl restriction endonuclease (Fig 1b. 2). This probe design allowed restriction digestion of $1^{\text {st }}$ generation RCPs without the addition of restriction oligonucleotides, and further resulted in RCP monomers that could be directly ligated on independent ligation barcodes. The efficiency of this novel RCP restriction digestion approach was evaluated, and compared to the 
conventional approach used for magnetic-bead based C2CA (Goransson et al. 2012). We achieved up to $100 \%$ efficiency using hairpin modified PLPs, indicating that the cleavage of $1^{\text {st }}$ generation RCPs and their ligation was successful, thereby validating our PLP design (Suppl. Fig. 2a). Additionally, this PLP design allowed to perform $1^{\text {st }}$ generation RCP restriction and monomer ligation simultaneously, as ligation barcodes do not contain the Sapl restriction site (Suppl. Fig. 2b), and also permitted to perform the protocol without enzyme inactivation, further simplifying the assay implementation since the ramping-up of temperature after incubation steps for enzyme inactivation, was not required (Suppl. Fig 2c).

Second, we adapted a previously described simple methodology for producing on-chip microarrays on unmodified polymer surfaces (Sun et al. 2012). The polymer microarray production was optimized and two main modifications were made to allow on-chip immobilized $2^{\text {nd }}$ generation RCPs (i) In contrast to the reported procedure by Sun et al.(Sun et al. 2012), the UV treatment was performed before and not after the oligonucleotide solution printing (Fig 4a), which allowed the elimination of signals observed outside the arrayed spots (Suppl. Fig 3). (ii) Substantially larger spots (500 $\mu \mathrm{m}$ in diameter) were printed on the $\mathrm{COC}$ surface using a pipetting robot and not a dedicated microarray printing instrument, which required optimization of the detergent (Triton-X) concentration in the spotting buffer, in order to obtain homogeneous spots (Suppl. Fig 3b). With the optimized microarray conditions, ligation barcodes immobilized on the COC surface could be used to circularize RCP monomers released from the beads, and prime the $2^{\text {nd }} \mathrm{RCA}$ reaction (Fig $4 \mathrm{~b}$ ). This enabled us to perform imaging and digital quantification of RCPs directly on- chip with regular fluorescence microscopy (Fig 4c).

To evaluate the analytical sensitivity of the developed $\mathrm{COC}$ arrays and generation of $2^{\text {nd }}$ generation RCPs on a surface, we designed two PLPs targeting a mutation in codon 12 of the oncogene KRAS, to detect the wildtype (GGT) and the mutant (GAT) variants. Each probe contained the common restriction hairpin-loop but independent labeling and ligation dual-barcodes (Table 1). This design resulted in $2^{\text {nd }}$ generation RCPs from each variant synthetized in separate spots on the array and detected with independently labeled barcode probes (Fig 4b). A PLP/C2CA assay, using a mix of mutant and wildtype specific PLPs, was performed with serial dilutions of synthetic mutant KRAS target, prepared at different ratios with the wild type target. Reaction steps on beads (Fig. 1b Steps 1-4) were performed off-chip and after RCA, beads were washed and re-suspended in the digestion/ligation reaction mix. This suspension was flowed over the arrays with subsequent on-chip monomer ligation, $2^{\text {nd }}$ RCA and labeling. RCPs were successfully digested, transferred from the magnetic beads and ligated to the barcodes immobilized on the $\mathrm{COC}$ array. The arrays displayed a dynamic range of 4 logs and it was possible to detect the mutated target down to a mutant: wildtype ratio of 1:1000 (0.1 fM initial target concentration) (Fig 4c-d).

By fully exploiting the reported multiplex capacity of PLPs (Hardenbol et al. 2005), in combination with the spatial analysis format of the microarray, and the use of RCPs as digital sequencing substrates (Drmanac et al. 2010), these microarrays have the potential to be developed into "multi-dimensional" arrays that allow simultaneous analysis of a large amount of DNA sequences, without requiring any compartmentalization for preparation, detection or read-out. 
a)

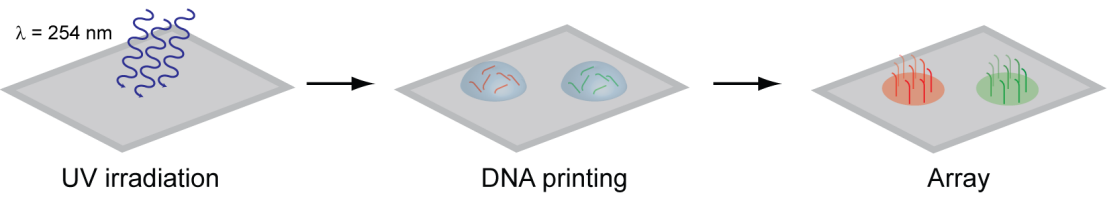

b)

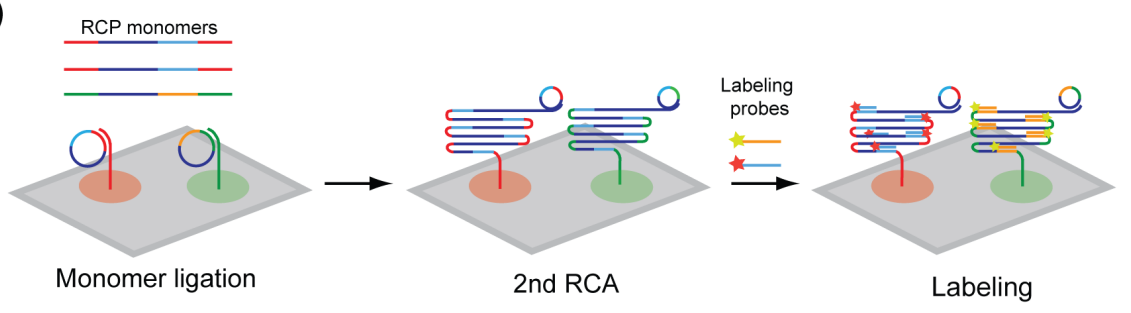

c)

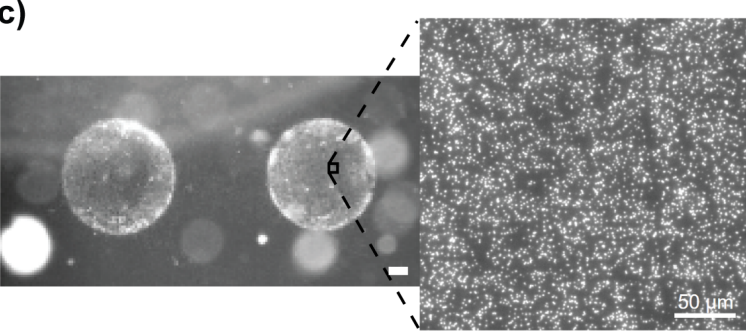

e) mutant
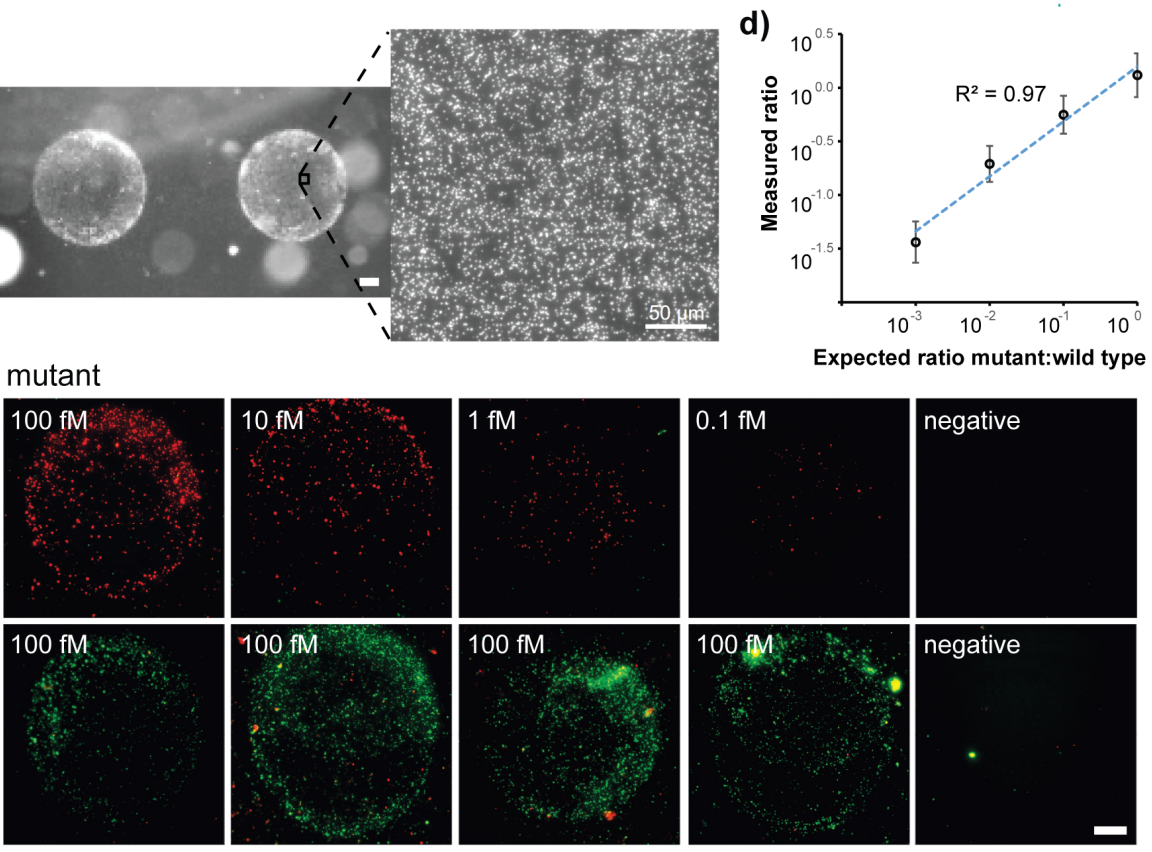

Expected ratio mutant:wild type

wildtype

Fig. $4 \mathrm{COC}$ based array for integrated on-chip digital read-out. a) Schematics of the microarray printing. COC slides are irradiated with UV light to activate the surface. Thereafter, dilutions of DNA oligonucleotides (ligation barcodes) containing (CT) 10 tags to the 5 ' end are printed with a pipetting robot on the activated surface, incubated and washed. b) Schematics of RCA amplification on COC arrays. RCP monomers released from the magnetic beads after on-bead steps of C2CA, are added to the arrays to be ligated on the immobilized ligation barcodes (red for mutant and green for wildtype). Circularized monomers are amplified by RCA and subsequently labeled with fluorescently-tagged barcode probes ( $\mathrm{Cy} 5$ for mutant, Сy3 for wildtype). e) Representative picture of a printed microarray under optimized conditions after RCA amplification, scale bar $=100 \mu \mathrm{m}$. Inset showing resulting RCPs in the polymer microarray that are imaged and counted digitally using regular fluorescence microscopy. d) KRAS mutant codon 12 (GAT) and wild type (GTT) synthetic targets were mixed at different ratios 1:1, 1:10, 1:100 and 1:1000 and were subjected to magnetic bead C2CA using a combination of PLPs targeting each variant and containing independent labeling and ligation barcodes. After the restriction reaction, resulting RCP monomers were processed as described in a). Error bars indicate standard deviation ( $n=2,8$ spots/target per chip). e) Representative pictures of array spots showing the results from the different target ratios used in d). Initial target concentrations are indicated. Scale bar $=100 \mu \mathrm{m}$ 


\subsection{Simple microfluidic chip for integrated DNA analysis in continuous flow}

Building on the developed modules and optimized functionalities, but without the ambition to develop at this stage a fully optimized and integrated system, we designed a simple prototype microfluidic chip. This allowed us to perform an initial proof-of-concept of an integrated magnetic bead based PLP/C2CA protocol in continuous flow mode, by combining the microfluidic fluidized bed principle with the developed polymer microarray. The designed microfluidic chip prototype was fabricated in $\mathrm{COC}$ and comprised a larger single rhombic microfluidic chamber, in which the fluidized bed was established at the low-angle $\left(13^{\circ}\right)$ entrance, and the microarray was positioned closer to the outlet (Fig 5a). With this simple design, it was possible to perform a complete PLP/C2CA protocol with solid-phase transfer (Fig $5 b$ ), from DNA target capture to imaging, with successful detection of RCPs on the microarrays (Fig. 5c) down to $1 \mathrm{pM}$ initial target concentrations. Further optimization in terms of design and operation will be needed to achieve optimal sensitivity. We hypothesize that during the solid phase transfer process (Fig $5 b$ ), the mass transport of monomers over the surface of the arrays is not optimal using this prototype design, and only a fraction of the released monomers reach the active surface of the array. A chamber geometry and flow rate conditions that take into account parameters for optimal mass transport, i.e Peclet numbers (Squires et al. 2008), could substantially improve upon this limitation to achieve the analytical sensitivity illustrated in Fig $4 \mathrm{e}$.

Despite this, our proof-of-concept demonstrates that a simple continuous-flow microfluidic DNA analyzer (sample-in $\rightarrow$ result-out) is possible, by the implementation of the microfluidic fluidized bed in combination with the polymer microarray. Normally, a continuous-flow microfluidic chip for such a complex multi-step protocol like PLP/C2CA, would require at least several chambers and multiple channels, including functionalities such as valves and passive mixers to achieve assay miniaturization. Additionally, the system must be connected to actuators such as motors necessary for mechanical magnet and valve actuation, therefore resulting in a highly complex and technically demanding system. Our proposed microfluidic chip, although still requiring further optimizations to achieve its maximum analytical capacity, is much simpler to fabricate and operate while showing the same processing capacity as more complex systems (Wu et al. 2013; Zhang and Da 2010). These features make this new chip principle attractive for the implementation of an integrated system for nucleic acid analysis for research or diagnostic applications. Moreover, this prototype chip, being fabricated in a polymer material as $\mathrm{COC}$, constitutes a simple, low-cost solution towards large-scale production of disposable polymer devices by injection molding. 
a)

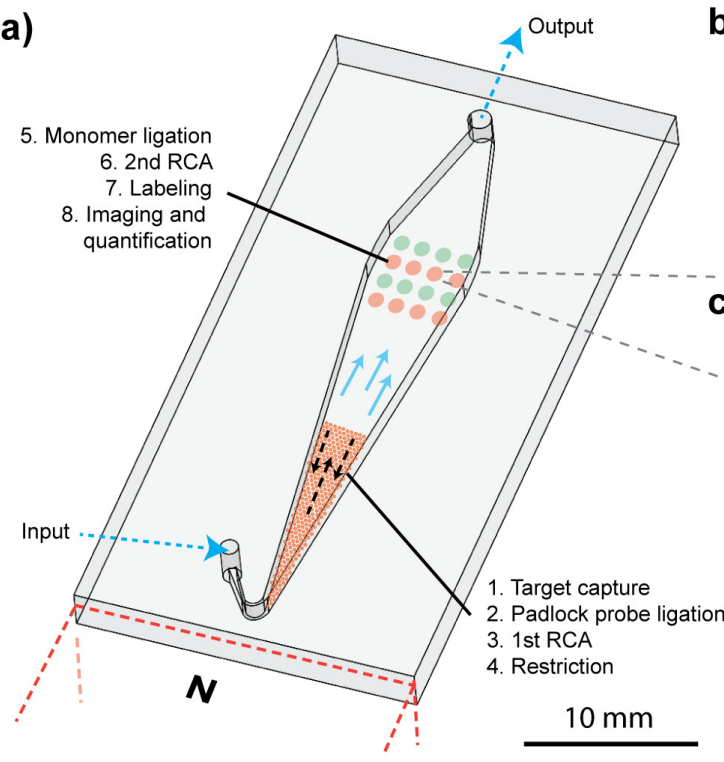

b)

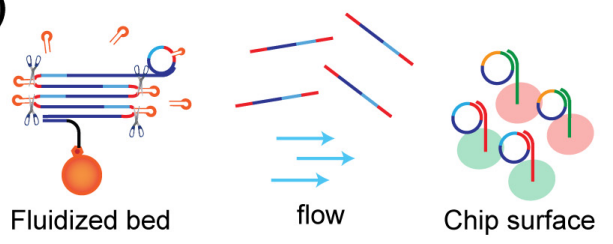

Fig. 5: Simple microfluidic chip prototype for complete DNA analysis using the fluidized bed approach. a) Illustration of the microfluidic chip design. It comprises a rhombic chamber similar to the one in Fig. 1a, but with a larger size to harbor the microarray in the wider part of the chamber. This allows for modular solid-phase transfer reactions where (i) the fluidized bed serves as a 3D dynamic support for efficient DNA target capture, sequence recognition by ligation of PLPs, RCA and restriction digestion and (ii) the microarray planar surface to produce immobilized RCPs that can be detected and quantified directly on-chip. b) Illustration of the solid phase transfer process where $1^{\text {st }}$ generation RCPs attached to the magnetic beads in the fluidized bed are released by restriction digestion, as the chip is operated in continuous-flow released monomers are transferred to the chip planar surface for simultaneous circularization on the grafted ligation barcodes in the microarray spots. c) Representative picture of array spots after performing the full PLP/C2CA protocol in the proposed microfluidic chip using an initial target concentration of $10 \mathrm{pM}$. Bottom right: Figure showing the number of RCPs/spot obtained for a series complete onchip experiments. Error bars indicate standard deviation $(n=2)$

\section{Conclusions}

Here, we demonstrated the use of a continuous-flow microfluidic system with a simplified passive mixing approach for DNA analysis. It is based on a fluidized bed principle for magnetic bead handling in microfluidic structures. This approach only requires a polymer microfluidic chip with a simple chamber geometry and a permanent magnet, which are easy to fabricate and operate. We evaluated the performance of this approach by testing the efficiency of different steps included in magnetic bead based PLP/C2CA, an assay for nucleic acid analysis consisting of a complex multi-step protocol that requires handling of magnetic beads and the use of various reagents, thus making it challenging for miniaturization with continuous-flow microfluidics. Here, we showed that a process like specific target capture by hybridization can be highly efficient using the fluidized bed, even when operated at high flow rates, owing to the hydrodynamic recirculation of beads. This feature allows to process large volumes in short times, a limitation often encountered in other platforms like digital or droplet microfluidics. We also demonstrated that the fluidized bed is efficient in performing common nucleic acid manipulation reactions, achieving superior performance compared to other types of magnetic actuation in continuous 
and stop-flow microfluidics. The fluidized bed offers a dynamic solid phase regime that optimizes mixing and contributes to overcome diffusion barriers, and avoids surface saturation leading to efficient molecular interactions, a critical parameter in biochemical reactions.

Moreover, to show the high potential of the microfluidic fluidized bed as a simple module to integrate in a $\mu$ TAS for DNA analysis, we combined this module with on-chip microarray detection. We first modified the design of the PLPs used in C2CA, to contain a hairpin-loop structure for a simplified restriction digestion scheme that enabled a surface transfer amplification protocol. This allowed us to obtain $2^{\text {nd }}$ generation RCPs on a surface, rather than in homogeneous solution, which requires either a dedicated instrument or off-chip microscope slide analysis for quantification. Moreover, we implemented and optimized a microarray based on hydrophobic unmodified polymer as a simple integrated read-out module of the surface-bound RCPs. The fabrication of this COC arrays was proven fast and simple, and we tested the analytical capabilities of these microarrays with an assay designed to detect a clinically relevant variant in the KRAS oncogene. The polymer microarrays displayed a dynamic range of 4 logs and allowed detection of the mutation down to a 1:1000 ratio and at concentrations as low as $0.1 \mathrm{fM}$.

Finally, with these established assay and read-out conditions, we provided the proof-of-concept of a simple microfluidic chip that integrates the fluidized bed with the polymer-based microarray for total nucleic acid processing. This combination drastically reduced the complexity of the microfluidic device and the instrumentation needed for actuation and analysis, allowing to perform a complex molecular multi-step protocol like PLP/C2CA within a simple single-chamber microfluidic chip in continuous-flow. After proper optimization in terms of chip design and setup, the proposed microfluidic chip can be developed to adapt to any of the applications reported for C2CA due to its high simplicity in terms of technical requirements for fabrication and operation. These include diagnosis of infectious diseases such as tuberculosis (Engstrom et al. 2013), urinary tract infections (Mezger et al. 2015a), detection of bio-security agents (Goransson et al. 2012), RNA viruses (Ke et al. 2011) or production of single stranded DNA libraries (Gu and Breaker 2013; Lohmann et al. 2007; Schmidt et al. 2015), .

\section{Acknowledgements}

The authors wish to thank Dr. Monica Brivio for initial conception of the project. Fluigent for providing a pressure system to Stockholm University and Microfluidic ChipShop for providing ChipGenie ${ }^{\circledR} \mathrm{P}$ and microfluidic chips. Lucile Alexandre for array and chip preparations and Dr. Narayanan Madaboosi for comments on the manuscript. M.N acknowledges FP7 funded CanDO project [FP7-610472] and SSF Flu-ID project [SBE13-0125]. I.P, JL.V and S.D acknowledge partial support from ANR project "Digidiag" [ANR 10-NANB-0002], EU projects ERCadg Cello [FP7-IDEAS-ERC-321107] and FP7 project NAPES [FP7-NMP-2013-SMALL-7]. D.F acknowledges the ARC foundation for young researcher fellowship.

\section{References}

Berensmeier, S., 2006. Magnetic particles for the separation and purification of nucleic acids. Appl Microbiol Biotechnol 73(3), 495-504. 
Dahl, F., Baner, J., Gullberg, M., Mendel-Hartvig, M., Landegren, U., Nilsson, M., 2004. Circle-to-circle amplification for precise and sensitive DNA analysis. Proc Natl Acad Sci U S A 101(13), 4548-4553.

Drmanac, R., Sparks, A.B., Callow, M.J., Halpern, A.L., Burns, N.L., Kermani, B.G., Carnevali, P., Nazarenko, I., Nilsen, G.B., Yeung, G., Dahl, F., Fernandez, A., Staker, B., Pant, K.P., Baccash, J., Borcherding, A.P., Brownley, A., Cedeno, R., Chen, L., Chernikoff, D., Cheung, A., Chirita, R., Curson, B., Ebert, J.C., Hacker, C.R., Hartlage, R., Hauser, B., Huang, S., Jiang, Y., Karpinchyk, V., Koenig, M., Kong, C., Landers, T., Le, C., Liu, J., McBride, C.E., Morenzoni, M., Morey, R.E., Mutch, K., Perazich, H., Perry, K., Peters, B.A., Peterson, J., Pethiyagoda, C.L., Pothuraju, K., Richter, C., Rosenbaum, A.M., Roy, S., Shafto, J., Sharanhovich, U., Shannon, K.W., Sheppy, C.G., Sun, M., Thakuria, J.V., Tran, A., Vu, D., Zaranek, A.W., Wu, X., Drmanac, S., Oliphant, A.R., Banyai, W.C., Martin, B., Ballinger, D.G., Church, G.M., Reid, C.A., 2010. Human genome sequencing using unchained base reads on self-assembling DNA nanoarrays. Science 327(5961), 78-81.

Duncombe, T.A., Tentori, A.M., Herr, A.E., 2015. Microfluidics: reframing biological enquiry. Nat Rev Mol Cell Biol 16(9), 554-567.

Engstrom, A., Zardan Gomez de la Torre, T., Stromme, M., Nilsson, M., Herthnek, D., 2013. Detection of rifampicin resistance in Mycobacterium tuberculosis by padlock probes and magnetic nanobead-based readout. PLoS One 8(4), e62015.

Ericsson, O., Jarvius, J., Schallmeiner, E., Howell, M., Nong, R.Y., Reuter, H., Hahn, M., Stenberg, J., Nilsson, M., Landegren, U., 2008. A dual-tag microarray platform for high-performance nucleic acid and protein analyses. Nucleic Acids Res 36(8), e45.

Ferraro, D., Champ, J., Teste, B., Serra, M., Malaquin, L., Viovy, J.L., de Cremoux, P., Descroix, S., 2016. Microfluidic platform combining droplets and magnetic tweezers: application to HER2 expression in cancer diagnosis. Sci Rep 6, 25540.

Gijs, M.A., Lacharme, F., Lehmann, U., 2010. Microfluidic applications of magnetic particles for biological analysis and catalysis. Chem Rev 110(3), 1518-1563.

Gijs, M.A.M., 2004. Magnetic bead handling on-chip: new opportunities for analytical applications. Microfluid Nanofluid 1(1), 22-40.

Goransson, J., Ke, R., Nong, R.Y., Howell, W.M., Karman, A., Grawe, J., Stenberg, J., Granberg, M., Elgh, M., Herthnek, D., Wikstrom, P., Jarvius, J., Nilsson, M., 2012. Rapid identification of bio-molecules applied for detection of biosecurity agents using rolling circle amplification. PLoS One 7(2), e31068.

Gu, H., Breaker, R.R., 2013. Production of single-stranded DNAs by self-cleavage of rolling-circle amplification products. Biotechniques 54(6), 337-343.

Hardenbol, P., Yu, F., Belmont, J., Mackenzie, J., Bruckner, C., Brundage, T., Boudreau, A., Chow, S., Eberle, J., Erbilgin, A., Falkowski, M., Fitzgerald, R., Ghose, S., lartchouk, O., Jain, M., Karlin-Neumann, G., Lu, X., Miao, X., Moore, B., Moorhead, M., Namsaraev, E., Pasternak, S., Prakash, E., Tran, K., Wang, Z., Jones, H.B., Davis, R.W., Willis, T.D., Gibbs, R.A., 2005. Highly multiplexed molecular inversion probe genotyping: over 10,000 targeted SNPs genotyped in a single tube assay. Genome Res 15(2), 269-275.

Hayes, M.A., Polson, N.A., Garcia, A.A., 2001. Active control of dynamic supraparticle structures in microchannels. Langmuir 17(9), 2866-2871.

Holubova, L., Knotek, P., Palarcik, J., Cadkova, M., Belina, P., Vlcek, M., Korecka, L., Bilkova, Z., 2014. Magnetic microparticles post-synthetically coated by hyaluronic acid as an enhanced carrier for microfluidic bioanalysis. Mat Sci Eng C-Mater 44, 345-351.

Hoshino, K., Huang, Y.-Y., Lane, N., Huebschman, M., Uhr, J.W., Frenkel, E.P., Zhang, X., 2011. Microchipbased immunomagnetic detection of circulating tumor cells. Lab on a Chip 11(20), 3449-3457.

Iranmanesh, I., Ohlin, M., Ramachandraiah, H., Ye, S., Russom, A., Wiklund, M., 2016. Acoustic microvortexing of fluids, particles and cells in disposable microfluidic chips. Biomed Microdevices 18(4), 71. 
Jarvius, J., Melin, J., Goransson, J., Stenberg, J., Fredriksson, S., Gonzalez-Rey, C., Bertilsson, S., Nilsson, M., 2006. Digital quantification using amplified single-molecule detection. Nat Methods 3(9), 725-727.

Julich, S., Hotzel, H., Gartner, C., Trouchet, D., Fawzy El Metwaly Ahmed, M., Kemper, N., Tomaso, H., 2016. Evaluation of a microfluidic chip system for preparation of bacterial DNA from swabs, air, and surface water samples. Biologicals 44(6), 574-580.

Julich, S., Kopinc, R., Hlawatsch, N., Moche, C., Lapanje, A., Gärtner, C., Tomaso, H., 2014. Lab-on-a-chip modules for detection of highly pathogenic bacteria: from sample preparation to detection. In: Cullum, M., McLamore, E.S. (Eds.), Smart Biomedical and Physiological Sensor Technology XI, p. 91070R.

Ke, R., Zorzet, A., Goransson, J., Lindegren, G., Sharifi-Mood, B., Chinikar, S., Mardani, M., Mirazimi, A., Nilsson, M., 2011. Colorimetric nucleic acid testing assay for RNA virus detection based on circle-to-circle amplification of padlock probes. J Clin Microbiol 49(12), 4279-4285.

Kojima, T., Takei, Y., Ohtsuka, M., Kawarasaki, Y., Yamane, T., Nakano, H., 2005. PCR amplification from single DNA molecules on magnetic beads in emulsion: application for high-throughput screening of transcription factor targets. Nucleic Acids Res 33(17), e150.

Kucerova, J., Svobodova, Z., Knotek, P., Palarcik, J., Vlcek, M., Kincl, M., Horak, D., Autebert, J., Viovy, J.L., Bilkova, Z., 2014. PEGylation of magnetic poly(glycidyl methacrylate) microparticles for microfluidic bioassays. Mater Sci Eng C Mater Biol Appl 40, 308-315.

Kuhnemund, M., Witters, D., Nilsson, M., Lammertyn, J., 2014. Circle-to-circle amplification on a digital microfluidic chip for amplified single molecule detection. Lab Chip 14(16), 2983-2992.

Lacharme, F., Vandevyver, C., Gijs, M.A., 2008. Full on-chip nanoliter immunoassay by geometrical magnetic trapping of nanoparticle chains. Anal Chem 80(8), 2905-2910.

Larsson, C., Koch, J., Nygren, A., Janssen, G., Raap, A.K., Landegren, U., Nilsson, M., 2004. In situ genotyping individual DNA molecules by target-primed rolling-circle amplification of padlock probes. Nat Methods 1(3), 227232.

Le Nel, A., Minc, N., Smadja, C., Slovakova, M., Bilkova, Z., Peyrin, J.-M., Viovy, J.-L., Taverna, M., 2008. Controlled proteolysis of normal and pathological prion protein in a microfluidic chip. Lab on a Chip 8(2), $294-301$.

Lizardi, P.M., Huang, X., Zhu, Z., Bray-Ward, P., Thomas, D.C., Ward, D.C., 1998. Mutation detection and single-molecule counting using isothermal rolling-circle amplification. Nature genetics 19(3), 225-232.

Lohmann, J.S., Stougaard, M., Koch, J., 2007. A new enzymatic route for production of long 5'-phosphorylated oligonucleotides using suicide cassettes and rolling circle DNA synthesis. BMC Biotechnol 7, 49.

Margulies, M., Egholm, M., Altman, W.E., Attiya, S., Bader, J.S., Bemben, L.A., Berka, J., Braverman, M.S., Chen, Y.J., Chen, Z., Dewell, S.B., Du, L., Fierro, J.M., Gomes, X.V., Godwin, B.C., He, W., Helgesen, S., Ho, C.H., Irzyk, G.P., Jando, S.C., Alenquer, M.L., Jarvie, T.P., Jirage, K.B., Kim, J.B., Knight, J.R., Lanza, J.R., Leamon, J.H., Lefkowitz, S.M., Lei, M., Li, J., Lohman, K.L., Lu, H., Makhijani, V.B., McDade, K.E., McKenna, M.P., Myers, E.W., Nickerson, E., Nobile, J.R., Plant, R., Puc, B.P., Ronan, M.T., Roth, G.T., Sarkis, G.J., Simons, J.F., Simpson, J.W., Srinivasan, M., Tartaro, K.R., Tomasz, A., Vogt, K.A., Volkmer, G.A., Wang, S.H., Wang, Y., Weiner, M.P., Yu, P., Begley, R.F., Rothberg, J.M., 2005. Genome sequencing in microfabricated highdensity picolitre reactors. Nature 437(7057), 376-380.

Mezger, A., Gullberg, E., Goransson, J., Zorzet, A., Herthnek, D., Tano, E., Nilsson, M., Andersson, D.I., 2015a. A general method for rapid determination of antibiotic susceptibility and species in bacterial infections. J Clin Microbiol 53(2), 425-432.

Mezger, A., Kuhnemund, M., Nilsson, M., Herthnek, D., 2015b. Highly specific DNA detection employing ligation on suspension bead array readout. N Biotechnol 32(5), 504-510.

Mezger, A., Ohrmalm, C., Herthnek, D., Blomberg, J., Nilsson, M., 2014. Detection of rotavirus using padlock probes and rolling circle amplification. PLoS One 9(11), e111874. 
Nilsson, M., Malmgren, H., Samiotaki, M., Kwiatkowski, M., Chowdhary, B.P., Landegren, U., 1994. Padlock probes: circularizing oligonucleotides for localized DNA detection. Science (New York, N.Y.) 265(5181), 20852088.

Pereiro, I., Bendali, A., Tabnaoui, S., Alexandre, L., Srbova, J., Bilkova, Z., Deegan, S., Joshi, L., Viovy, J.L., Malaquin, L., Dupuy, B., Descroix, S., 2017a. A new microfluidic approach for the one-step capture, amplification and label-free quantification of bacteria from raw samples. Chem Sci 8(2), 1329-1336.

Pereiro, I., Tabnaoui, S., Fermigier, M., du Roure, O., Descroix, S., Viovy, J.L., Malaquin, L., 2017b. Magnetic fluidized bed for solid phase extraction in microfluidic systems. Lab Chip 17(9), 1603-1615.

Rida, A., Gijs, M.A., 2004. Manipulation of self-assembled structures of magnetic beads for microfluidic mixing and assaying. Anal Chem 76(21), 6239-6246.

Sackmann, E.K., Fulton, A.L., Beebe, D.J., 2014. The present and future role of microfluidics in biomedical research. Nature 507(7491), 181-189.

Saliba, A.E., Saias, L., Psychari, E., Minc, N., Simon, D., Bidard, F.C., Mathiot, C., Pierga, J.Y., Fraisier, V., Salamero, J., Saada, V., Farace, F., Vielh, P., Malaquin, L., Viovy, J.L., 2010. Microfluidic sorting and multimodal typing of cancer cells in self-assembled magnetic arrays. Proc Natl Acad Sci U S A 107(33), 14524-14529.

Schmidt, T.L., Beliveau, B.J., Uca, Y.O., Theilmann, M., Da Cruz, F., Wu, C.T., Shih, W.M., 2015. Scalable amplification of strand subsets from chip-synthesized oligonucleotide libraries. Nat Commun 6, 8634.

Squires, T.M., Messinger, R.J., Manalis, S.R., 2008. Making it stick: convection, reaction and diffusion in surface-based biosensors. Nat Biotechnol 26(4), 417-426.

Sun, Y., Perch-Nielsen, I., Dufva, M., Sabourin, D., Bang, D.D., Hogberg, J., Wolff, A., 2012. Direct immobilization of DNA probes on non-modified plastics by UV irradiation and integration in microfluidic devices for rapid bioassay. Anal Bioanal Chem 402(2), 741-748

Tamanaha, C.R., Mulvaney, S.P., Rife, J.C., Whitman, L.J., 2008. Magnetic labeling, detection, and system integration. Biosens Bioelectron 24(1), 1-13.

Teste, B., Jamond, N., Ferraro, D., Viovy, J.L., Malaquin, L., 2015. Selective handling of droplets in a microfluidic device using magnetic rails. Microfluidics and Nanofluidics 19(1), 141-153.

Wang, Y., Zhao, Y., Cho, S.K., 2007. Efficient in-droplet separation of magnetic particles for digital microfluidics. J Micromech Microeng 17(10), 2148-2156.

Wu, J., Kodzius, R., Cao, W., Wen, W., 2013. Extraction, amplification and detection of DNA in microfluidic chipbased assays. Microchimica Acta 181(13-14), 1611-1631.

Yaralioglu, G.G., Wygant, I.O., Marentis, T.C., Khuri-Yakub, B.T., 2004. Ultrasonic mixing in microfluidic channels using integrated transducers. Anal Chem 76(13), 3694-3698.

Zhang, C., Da, X., 2010. Single-Molecule DNA Amplification and Analysis Using Microfluidics. Chemical Reviews 110(8), 4910-4947. 


\section{Microfluidic magnetic fluidized bed for DNA analysis in continuous flow mode}

Iván Hernández-Neutaa*, lago Pereiro ${ }^{b, c, d *}$, Annika Ahlford ${ }^{a}$, Davide Ferraro ${ }^{b, c, d}$, Qiongdi Zhang ${ }^{b, c, d}$, Jean-Louis Viovy $^{b, c, d}$, Stéphanie Descroix ${ }^{b, c, d * *}$ and Mats Nilsson ${ }^{a \star *}$

a Science for Life Laboratory, Department of Biochemistry and Biophysics, Stockholm University, Stockholm Sweden

b Laboratoire Physico Chimie Curie, Institut Curie, PSL Research University, CNRS UMR168, 75005, Paris, France

c Sorbonne Universités, UPMC Univ Paris 06, 75005, Paris, France

dInstitut Pierre-Gilles de Gennes, 75005, Paris, France

${ }^{*}$ Authors contributed equally to this work

${ }^{* *}$ Corresponding authors

\section{Supplementary information}

Suppl. Fig.1. Aggregation of the magnetic beads after long incubation steps.

Suppl. Fig.2: Hairpin-loop modified PLPs allow to combine steps and remove enzyme inactivation.

Suppl. Fig.3: Optimization of the conditions for the production of unmodified polymer-based microarray for RCP quantification. 
a) After functionalization

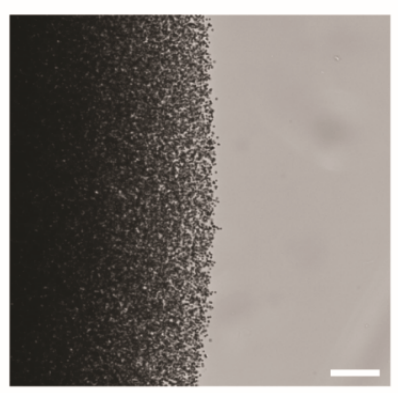

b)

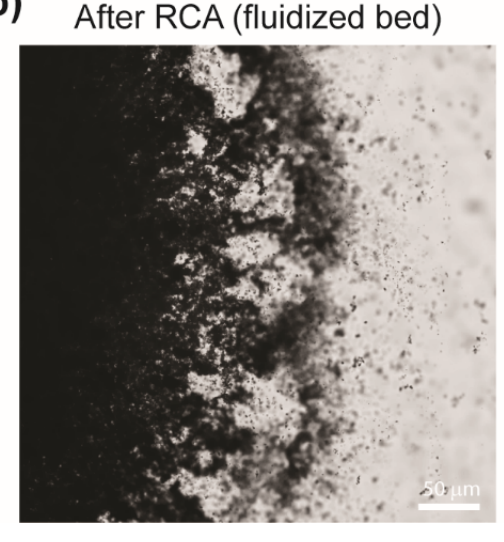

After RCA incubation (tube) 7

$(+) B S A,(+)$ polymerase

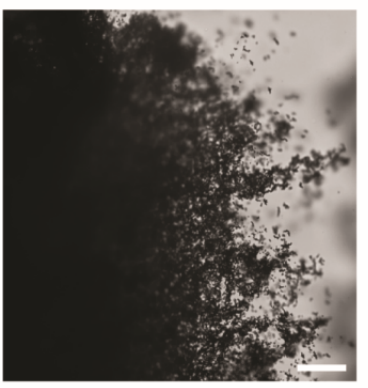

(-) BSA, (-) polymerase

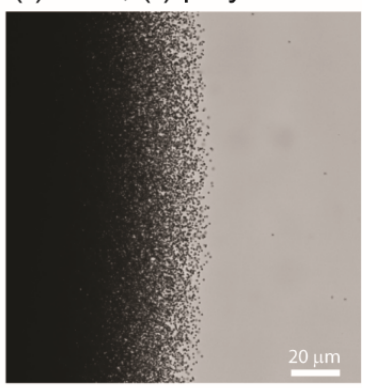

After RCA (ChipGenie)

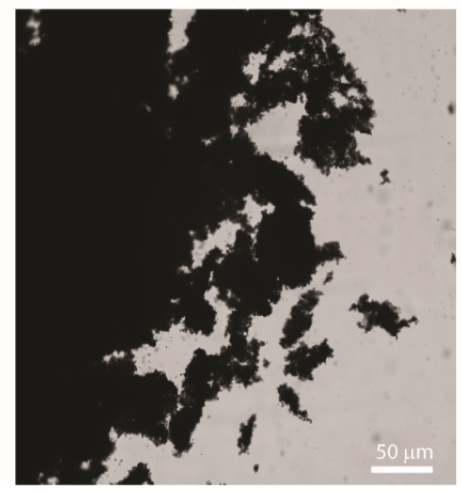

Suppl. Fig.1. Aggregation of the magnetic beads after long incubation steps. a) Beads aggregate after RCA reaction. Magnetic particles were collected and put in a microscope slide with the help of a magnet, a cover slip is put on top of the collected beads and the magnet is taken-off. This was done after bead functionalization (before any reaction), after RCA and after incubation in the RCA reaction buffer without BSA, enzymes, DNA or dNTPs. b) Pictures showing the size of the clusters after an RCA performed on the fludized bed (left) and the ChipGenie (right). Scale bars: $20 \mu \mathrm{m}$ (a) and $50 \mu \mathrm{m}$ (b) 
a)

Hairpin-loop based RCP digest

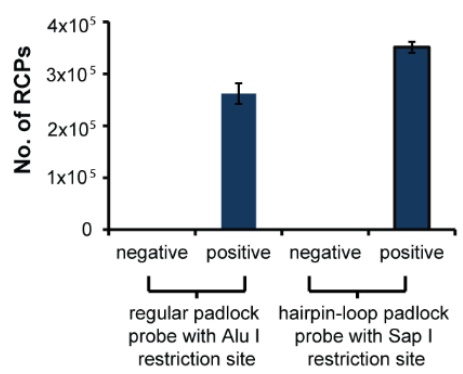

b) Combination of digestion and ligation

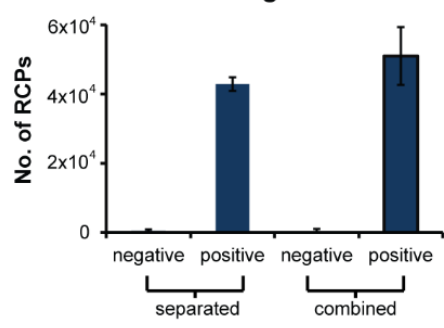

c)

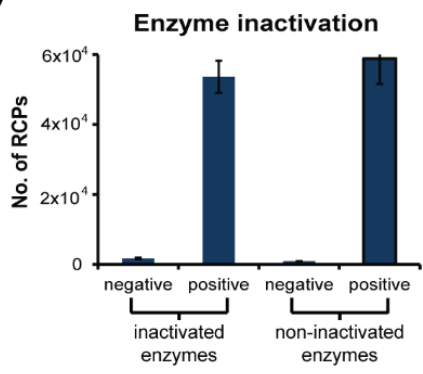

Suppl. Fig.2: Hairpin-loop modified PLPs allow to combine steps and remove enzyme inactivation. a) Evaluation of efficiency of C2CA using regular vs hairpin-loop modified PLPs. Regular C2CA utilizes PLPs containing an Alul restriction site. After $1^{\text {st }} \mathrm{RCA}$ a restriction oligonucleotide is added forming the double stranded substrate for the restriction digestion. Hairpin-loop modified PLP contain a hairpin-loop structure containing the double stranded substrate with the restriction site for Sapl, so the RCP restriction reaction is performed without addition of new oligonucleotides. b) The use of hairpin-loop PLP enabled the combination of digestion and ligation steps as RCP monomers are ligated in independent oligonucleotides (ligation barcodes) that do not contain the Sapl restriction site. c) Test of inactivated vs non-inactivated enzymes. An experiment where the enzymes up to RCP monomer circularization were not inactivated is compared to inactivation after single steps. All figures are results from RCP counting by ASMD quantification after complete C2CA reactions. Error bars correspond to standard deviation ( $\mathrm{n}$ $=2)$. 
a)

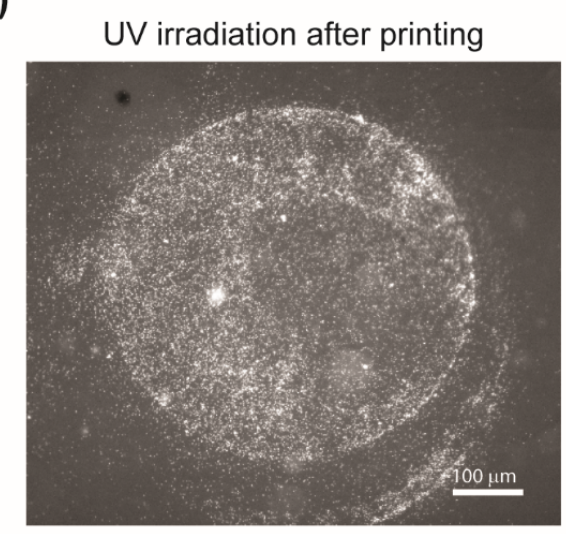

b)

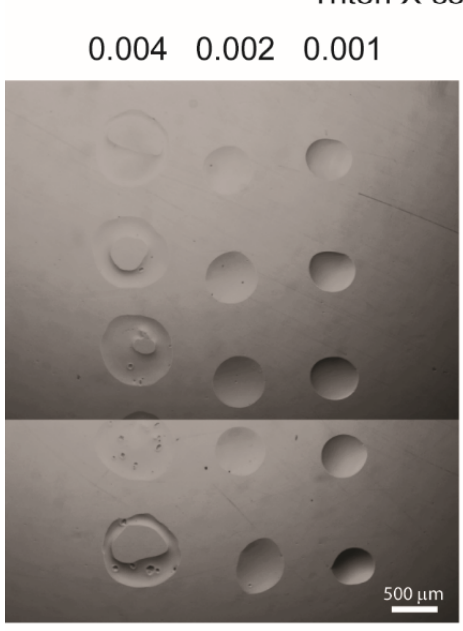

Bright field

\section{Triton $\mathrm{X}$ concentration (\%)}
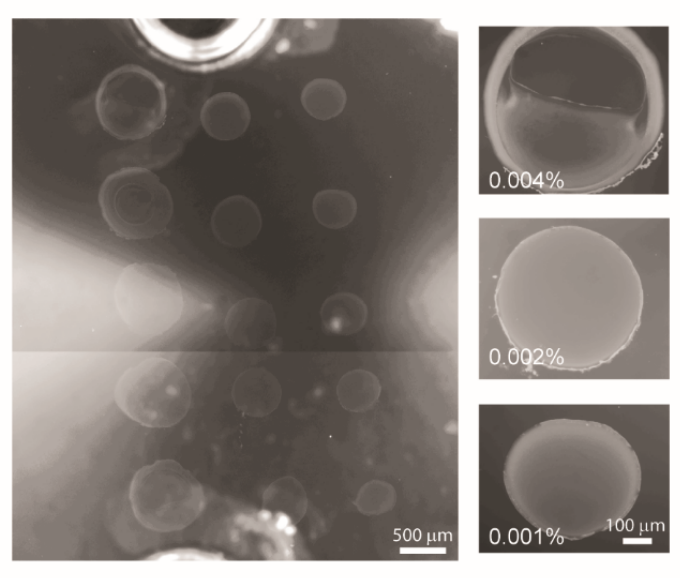

Cy3 channel

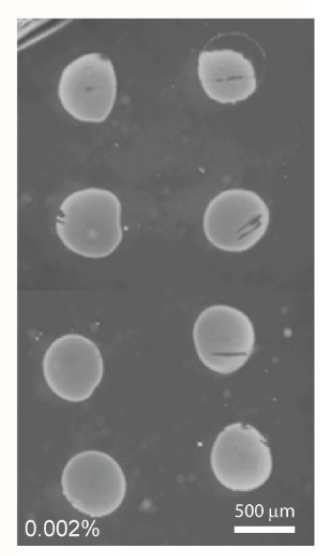

Suppl. Fig.3: Optimization of the conditions for the production of unmodified polymer-based microarray for RCP quantification. a) Comparison of arrays with UV irradiation after vs before printing of the oligonucleotides. b) Titration of the Triton- $X$ concentration in the printing buffer for homogeneous spots. Hybridization of a Cy3-tagged oligonucleotide complementary to the printed ligation template was used for these experiments. 